Revue d'histoire de l'Amérique française

REVUE D.HISTOIRE DE L'AMÉRIQUE FRANÇAISE

\title{
Essai sur les finances canadiennes (1700-1750) (suite)
}

\section{Guy Frégault}

Volume 12, numéro 4, mars 1959

URI : https://id.erudit.org/iderudit/301932ar

DOI : https://doi.org/10.7202/301932ar

Aller au sommaire du numéro

Éditeur(s)

Institut d'histoire de l'Amérique française

ISSN

0035-2357 (imprimé)

1492-1383 (numérique)

Découvrir la revue

Citer cet article

Frégault, G. (1959). Essai sur les finances canadiennes (1700-1750) (suite). Revue d'histoire de l'Amérique française, 12(4), 459-484.

https://doi.org/10.7202/301932ar d'utilisation que vous pouvez consulter en ligne.

https://apropos.erudit.org/fr/usagers/politique-dutilisation/ 


\title{
ESSAI SUR LES FINANCES CANADIENNES *
}

\section{(1700-1750)}

\author{
(suite)
}

\section{RECETTES ET DEPENSES AVANT 1728}

Puisque les états de prévision n'ont, en somme, que des rapports assez éloignés avec les dépenses du gouvernement du Canada, l'analyse que nous en avons faite nous a simplement mis au courant de certaines habitudes administratives de la France dans le domaine des finances coloniales; habitudes qui ne manquent pas de sens: ne nous laissent-elles pas entrevoir l'état d'esprit qu'une métropole économe entretient à l'égard de son aile américaine ? Une étude des recettes et des dépenses de la colonie nous donnera l'occasion d'approfondir cet état d'esprit.

Les deux questions auxquelles il nous faut chercher des réponses sont aussi importantes l'une que l'autre: que dépense le gouvernement canadien, et à quoi sa caisse s'alimente-t-elle ? Comme les recettes du pays ne sont pas constantes, que ses dépenses sont variables et que les circonstances de son évolution financière sont diverses, il devient nécessaire de répartir sur certaines périodes l'examen de ces questions fondamentales. Trois périodes se découpent d'elles-mêmes: la première s'étend du début du siècle à 1728 et correspond à la première phase de l'histoire de

* Voir, pour début de cet article, notre Revue, XII : 307-322. 
la monnaie de cartes; la deuxième commence en 1729 et englobe la seconde partie de la paix de trente ans; la troisième a pour considérer que les chiffres tirés des bordereaux de recette et de ligne de départ l'époque d'hostilités qu'inaugure, en 1744, la guerre de la Succession d'Autriche.

La caisse de la colonie s'alimente à deux sources: les sommes prévues par l'état du roi et les recettes extraordinaires. A ne considérer que les chiffres tirés des bordereaux de recette et de dépense, un équilibre surprenant apparaît entre celle-ci et celle-là (tableau II). Sur les dix-neuf années à propos desquelles il est possible d'avoir des renseignements dans notre première période, il n'y en aurait que huit où un excédent de dépenses se manifeste, et encore celui-ci ne dépasse-t-il que deux fois 100.000 livres. Les « recettes extraordinaires» constituent le grand facteur d'équilibre. Légèrement supérieures, pour autant qu'on les connaisse, à 50.000 livres par année entre 1705 et 1713 , elles atteignent la moyenne élevée de 154.500 livres de 1714 à 1719 , pour redescendre au-dessous de 100.000 livres (exactement 92.099) de 1720 à 1724 et se hausser au niveau de 116.000 livres pour les quatre dernières années de la période. Au cours de celle-ci prise dans son entier, elles comptent, année commune, pour 108.870 livres. Ainsi, la moyenne obtenue entre 1714 et 1719 ressort nettement comme exceptionnelle. A quoi cela tient-il ? A ce qu'en 1716 et en 1717, l'Etat réalise des gains, au moment de la réduction de la monnaie de cartes à $50 \%$ de sa valeur nominale, en faisant des paiements en cartes non réduites. Ces gains se chiffrent par 112.673 livres la première année et par 139.125, la seconde. $^{32}$ Sans cette spéculation heureuse (pour lui), le gouvernement colonial n'aurait, au cours de ces six ans, encaissé en moyenne que 112.555 livres en recettes extraordinaires, ce qui n'eût guère dépassé la moyenne de toute la période.

32 «Bordereau de la recette et depense faites en la Nouvelle-france, pendant l'année 1716, suivant le Compte arresté au $\mathrm{Sr}$ Petit Commis de

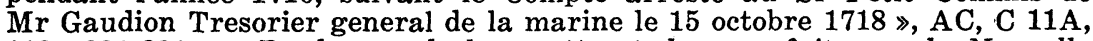
113: 286-286v; "Bordereau de la recette et depense faites en la Nouvelle france pendant l'année 1717. suivant le compte arresté au Sr Petit Commis de Mr Moufle de Champigny Tresorier general de la marine le 9. octobre $1719 »$, ibid., 305-305v. 
TABLEAU II

\section{RECETTES ET DÉPENSES 1705-1728}

\begin{tabular}{lcccc} 
Année & $\begin{array}{c}\text { Etat du roi } \\
\text { (livres) }\end{array}$ & $\begin{array}{c}\text { Recettes } \\
\text { extraordinaires } \\
\text { (livres) }\end{array}$ & $\begin{array}{c}\text { Total des } \\
\text { recettes } \\
\text { (livres) }\end{array}$ & $\begin{array}{c}\text { Dépenses } \\
\text { (livres) }\end{array}$ \\
1705 & 326.255 & 50.620 & 376.875 & 376.861 \\
1711 & 388.632 & 56.633 & 445.265 & 445.265 \\
1712 & 463.188 & 57.964 & 520.882 & 567.348 \\
1713 & 391.188 & 51.160 & 442.348 & 445.455 \\
1714 & 375.372 & 109.339 & 484.711 & 556.179 \\
1715 & 414.298 & 133.948 & 548.246 & 548.243 \\
1716 & 318.292 & 184.375 & 502.667 & 502.672 \\
1717 & 322.522 & 260.757 & 583.279 & 583.125 \\
1718 & 281.763 & 92.411 & 374.174 & 374.198 \\
1819 & 285.107 & 145.697 & 430.804 & 430.804 \\
1720 & 295.206 & 86.293 & 381.499 & 381.499 \\
1721 & 309.543 & 96.857 & 406.400 & 577.932 \\
1722 & 314.114 & 91.332 & 405.446 & 406.158 \\
1723 & 318.403 & 93.677 & 412.080 & 412.092 \\
1724 & 291.341 & 92.335 & 383.676 & 383.670 \\
1725 & 289.696 & 103.881 & 393.577 & 393.594 \\
1726 & 289.494 & 107.293 & 396.787 & 398.150 \\
1727 & 308.156 & 107.147 & 415.203 & 416.897 \\
1728 & 373.152 & 146.220 & 519.372 & 751.098 \\
\hline & & & &
\end{tabular}

En raison de l'importance du rôle stabilisateur que jouent les recettes extraordinaires, il faut nous demander de quoi elles sont faites. L'élément principal en est la vente, effectuée à divers particuliers, d'effets tirés des magasins du roi. Il est juste que cette vente soit inscrite en recette. Lorsque l'État métropolitain garnit les entrepôts de Québec, il porte au débit du compte de la colonie le prix des fournitures qu'il y fait expédier; quand, à son tour, l'Etat colonial vend ces articles aux Canadiens, l'intendant porte naturellement en recette les sommes qu'il en tire. Ainsi, il existe toujours dans les magasins du roi, à Québec, à Montréal et aux Trois-Rivières, un fonds de marchandises dont 
la métropole assure le maintien et à même lequel les habitants s'approvisionnent - de sel, par exemple, ou de poudre - selon leurs besoins et leurs moyens. Car le roi réalise un profit sur ces ventes. La Cour s'y attend: quand, en 1731, Maurepas dit escompter un excédent de recette, un des motifs de son optimisme est la prévision d'un surcroît de bénéfices sur les ventes des magasins..$^{33}$ Deux ans plus tard, Hocquart doit lui expliquer qu'il faut compter pour peu de chose le profit ainsi obtenu et surtout ne pas espérer que ce dernier puisse compenser les dépenses que Versailles refuse de prévoir: "Ce benefice peut, a la verité, remplacer les consommations qui se font [des effets des magasins], mais non suppleer aux excedents de plusieurs chapitres de Depenses employez dans l'estat du Roy. ${ }^{34}$

Nous pouvons maintenant voir, à l'aide de quelques exemples, en quoi consistent les recettes extraordinaires du gouvernement canadien. En 1711, elles s'établissent, nous le savons, à 56.633 livres, dont 53.067 proviennent des ventes des magasins; le reste est formé par la retenue régulière de six deniers pour livre $(2.5 \%)$ que le roi prélève sur la solde des militaires pour leur habillement. ${ }^{35}$ En 1714, les ventes des magasins constituent à elles seules toutes les recettes extraordinaires: 93.787 livres à Québec, 2.372 aux Trois-Rivières et 15.240 à Montréal.36 En 1715, sur des recettes extraordinaires de 133.948 livres, 118.405 représentent le produit des ventes du roi; 15.000 viennent du Domaine d'Occident: c'est une subvention appliquée au rétablissement du palais de l'intendant, incendié en 1713 ; restent 543 livres, qui proviennent d'exercices précédents. ${ }^{37}$ Ces quelques chiffres indiquent la place que les ventes des magasins occupent dans les recettes extraordinaires.

On serait porté à conclure tout de suite que ces dernières portent les revenus de l'Etat colonial au niveau relativement

\footnotetext{
33 Voir plus haut, note 12.

34 Hocquart à Maurepas, 24 octobre 1733, AC, C 11A, 60: 167.

35 « Exercice de 1711. Balance des fonds remis, Recettes Extraordinaires et des Despenses de L'année 1711 》, AC, C 11A, 113: 262.

36 \& Bordereau de la recette et depense faite en la Nouvelle france pendant l'année 1714», AC, C 11A, 113: 269.

37 \& Canada 1715. Balance des fonds remis, recettes extraordres et des depenses en acquits de l'année $1715 \gg, \mathrm{AC}, \mathrm{C} 11 \mathrm{~A}, 113$ : 279.
} 
élevé de ses dépenses. Pourtant, à parcourir la correspondance officielle de la première partie du XVIII ${ }^{e}$ siècle, on a l'impression que la situation financière du Canada est beaucoup plus sombre et singulièrement plus compliquée que ne le laissent croire les données que nous venons d'étudier. C'est que ces données n'expriment pas toute la vérité. Elles restent silencieuses sur deux points. En premier lieu, elles ne font pas état des dépenses rejetées d'un exercice sur l'autre. Ensuite, elles ne tiennent pas compte des dettes que l'Etat contracte envers ses fournisseurs et qu'il solde tant bien que mal avec de la monnaie fiduciaire. Examinons rapidement ces deux problèmes.

\section{Excédents de dépenses}

En 1727, assiégé par les embarras que lui suscitent une caisse à peu près vide et la perspective d'une caisse encore plus dégarnie l'année suivante, l'intendant Dupuy veut savoir la cause de cette situation intolérable et, pour en avoir le cœur net, il compulse tous les comptes du trésor sur lesquels il peut mettre la main. Il parvient à en rassembler une série continue depuis 1711. Cette année-là, rien de mieux équilibré en apparence que le compte du trésorier: recette de $\mathbf{4 4 5 . 2 6 5}$ livres, dépense de 445.265 livres. Attention! Le premier article de dépense se présente ainsi : excédent des dépenses de 1710, employé au compte de 1711 : 126.696 livres; pour payer cette somme, il a fallu anticiper sur les fonds faits par l'état du roi pour 1712. Aussi, l'année suivante, le premier article de dépense est-il constitué par une somme de 183.781 livres représentant l'excédent des dépenses de 1710 et de 1711 . Comment solder pareil excédent? En anticipant encore sur l'exercice de 1713 , où le déficit hérité de l'année précédente se chiffre par 145.667 livres, soit environ 100.000 livres de plus que ce qu'avoue notre tableau. Pour 1713, celui-ci ne retient qu'un excédent de dépense de 3.000 livres; un examen du compte de 1714 révèle toutefois qu'on y a reporté une dépense de 130.667 livres effectuée, en 1713, en sus des 445.000 livres inscrites au compte. En 1715, une augmentation soudaine des ventes des magasins - c'est la seconde en deux ans - permet d'absorber les déficits légués par l'exercice précédent. 
Le défilé des déficits reprend tout de suite après cette pause trop brève. En 1716, le trésorier reporte sur 1717 un excédent de dépenses de 46.000 livres. En 1717, bien que les ventes des magasins du roi accusent une augmentation de 50.000 livres sur l'année précédente, l'exercice se clôt quand même sur un autre excédent de dépenses (41.000 livres) dont on charge l'exercice suivant. Rappelons que si le gouvernement canadien n'avait pas spéculé sur la monnaie de cartes en 1716 et en 1717, il y aurait lieu d'ajouter quelque 250.000 livres à sa dette. En 1718, le déficit rejeté sur 1719 se chiffre par 113.000 livres. Il continue d'en aller ainsi, avec des hauts et des bas, au cours de toutes les années suivantes. Dans le compte de 1725 , il faut employer la somme de 103.000 livres, au premier article de dépense, pour payer le déficit de 1724. En octobre 1727, on voit que, pour fonctionner, le gouvernement canadien a dû tirer pour près de 200.000 livres de lettres de change sur les fonds de 1728 qui, bien entendu, ne sont pas encore ordonnés. Dupuy en conclut que le roi ne pourra pas se dispenser de faire « un remplacement tres considerable dans l'etat de 1728 sur l'excedent des depenses des années anterieures ».

En réalité, la situation est encore plus sombre que Dupuy ne la représente. On s'explique pourquoi dès qu'on se rappelle que l'intendant n'a pas vu le détail des comptes de 1726, de 1727 et de 1728. Au courant de tout, le commissaire Clairambault d'Aigremont trouve l'État colonial si fortement endetté qu'il estime urgent d'en rétablir le crédit. Certaines de ses obligations remontent assez loin en arrière. Ainsi, les dépenses effectuées depuis 1713 pour reconstruire le palais de l'intendant excèdent de $\mathbf{2 6 . 0 0 0}$ livres les sommes accordées à cet effet par la Cour. Jusqu'à 1725, on a dépensé pour les fortifications 34.881 livres de plus que les fonds ordonnés. Pour remplacer cette somme, Maurepas a prescrit de prendre 35.000 livres sur l'impôt prélevé à Montréal pour l'érection d'une enceinte autour de cette ville; mais les Montréalais mettent tant de mauvaise volonté à satisfaire le fisc qu'en 1728 ces 35.000 livres ne sont pas encore perçues. Ce ne sont là que des exemples. Selon un état abrégé que dresse Aigremont, les dépenses du Canada pour 1726, 1727 et 1728 - 
y compris l'excédent de 1725 rejeté sur 1726 et celui de 1728 rejeté sur 1729 - montent à 1.709 .999 livres et les fonds remis pour y faire face, à 1.196.631. Le total des excédents de dépenses atteint donc 513.368 livres. Ces dépenses sont faites. Comment le gouvernement canadien y a-t-il pourvu ? Par des ordonnances de paiement. Outre que ce papier ne se négocie pas aisément, sa multiplication est la preuve de la gêne financière de l'État. Voilà deux motifs qui engagent les négociants à exiger de lui des prix plus élevés que s'ils lui vendaient au comptant; voilà aussi qui explique en partie pourquoi sa dette fait boule de neige. Pour enrayer ce mouvement, Aigremont fait tirer sur les trésoriers généraux de la Marine à Paris pour 512.494 livres de lettres de change, ce qui le met en mesure de retirer les ordonnances de paiement répandues dans le commerce. Son dessein, explique-t-il, est « de conserver la confiance pour celles [ordonnances] qu'on sera obligé d'expedier pour les depenses absolument necessaires jusqu'a l'arrivée du Vaisseau du Roy l'année prochaine [1729], sans quoy la moitié du peuple seroit mort de faim ». Il ajoute cette considération ... humanitaire: «Et cela auroit causé un derangement tres facheux dans la colonie. ${ }^{38}$

A Paris, les comptables de la Marine se mettent au travail. Leur vue est de réduire autant que possible, sur papier, les excédents de dépenses de la lointaine colonie. Après avoir savamment jonglé avec les chiffres, ils concluent qu'Aigremont a omis dans ses bordereaux pour près de 115.000 livres de recettes. Les excédents de dépenses ne s'en établissent pas moins, selon leurs calculs, à environ 400.000 livres. D'où proviennent-ils ? Dans les seules années 1726,1727 et 1728 , le gouvernement canadien a acheté dans la colonie pour $\mathbf{5 5 2 . 5 9 7}$ livres de vivres et de munitions. Comme les états du roi n'avaient prévu à ce chapitre qu'une dépense de 120.508 livres, il en résulte un déficit de 432.089 livres. ${ }^{39}$ Ainsi, la plus grande partie des fonds que le roi n'ordon-

38 Aigremont à Maurepas, 8 novembre 1728, AC, C 11A, 113: 345-349. Voir * Dépenses du Canada suivant les Bordereaux et Balance envoyés par M. D'Aigremont », ibid., 339-344v; «Fonds de Canada. Fonds a faire a la Colonie 》, ibid., 444-444v. $349 \mathrm{v}-356$.

39 « Examen du Bordereau general des depenses», AC, C 11A, 113 : 
ne pas, mais que ses représentants dépensent quand même, se retrouvent, au bout du compte, entre les mains des commerçants du pays. En d'autres termes, l'Ettat colonial, qui vend à profit certaines marchandises aux particuliers, achète de ceux-ci beaucoup plus qu'il ne leur vend, et c'est là la principale cause de ses déficits annuels.

La monnaie de cartes: avant 1700

Il faut maintenant considérer l'autre problème auquel nous avons fait allusion plus haut: la dette de l'État canadien exprimée en monnaie fiduciaire et, singulièrement, en monnaie de cartes. Il ne s'agit pas, encore une fois, de reprendre tout le travail de Shortt. ${ }^{40}$ Bornons-nous à indiquer les rapports qui existent entre les émissions de cartes et les dépenses du gouvernement canadien.

Lorsque l'intendant de Meulles lance la première émission de cartes, en 1685, il le fait "Veu le manque de fonds de Sa $\mathrm{Ma}^{\text {t6 }}$ et la nécessité d'argent ou nous nous sommes trouvé pour entretenir et faire subsister les trouppes » et il s'engage à racheter toutes les cartes à leur valeur nominale au moyen \& des premiers deniers que $\mathrm{Sa} \mathrm{Ma}^{\text {té }}$ nous envoyera infailliblement par les vaisseaux de la présente année ». Trois mois plus tard, les fonds attendus sont arrivés; l'intendant invite les détenteurs de cartes à les lui rapporter pour recevoir, en échange, des sommes correspondantes de monnaie métallique. ${ }^{41}$ C'était là un expédient inusité. A son chef, de Meulles confia qu'il n'avait eu recours à ce procédé qu'à l'extrémité, «ne scachant plus a quel Saint [se] vouer $\gg .{ }^{42}$ Seignelay, du reste, le désapprouva. ${ }^{43}$ Une autre émission de cartes fut néanmoins effectuée en 1686. Rachetée six mois plus tard, elle était du même type que la première. ${ }^{44}$ En 1690 , nouvelle fabrication de cartes, suivie d'une autre encore, en 1691. Elles ont ceci de commun avec les émissions précédentes

\footnotetext{
41 Ordonnances du 8 juin et du 5 septembre 1685, ibid., 68-72.

42 Lettre du 24 septembre 1685, ibid., 74.

43 \& Extrait des reponses aux lettres receues du Canada pendant la presente année $1686 \gg$, ibid., 78 .

44 Ibid., 74-76, 80 .
} lxxxviii.

40 Voir l'introduction à ses Documents relatifs à la monnaie, 1: xxxii- 
qu'elles servent simplement à pourvoir aux «depenses depuis le commencement de chaque année jusqu'à l'arrivée des vaisseaux $»$. Il ne s'agit donc pas encore de dépenses faites par anticipation sur les fonds de l'année suivante.

Voici, cependant, qu'une autre évolution se dessine. La même année 1691, voulant éviter de lancer d'autres cartes sur le marché, Champigny prend sur lui d'ordonner au trésorier de la colonie de tirer 200.000 livres de lettres de change sur les trésoriers généraux de la Marine, payables en mai 1692 «sur les fonds qui seront ordonnez pour ce pays »; il distribue ces traites aux négociants du Canada, qui lui remettent en échange une somme correspondante de monnaie: argent qui lui permettra de faire face aux besoins de l'État. "Je vous prie, écrit-il à Pontchartrain, d'ordonner le payement des dittes lettres à leur échéance affin que nous puissions nous servir de pareils expediens à l'avenir sy vous le jugez a propos $\$ .45$ Ici, on se trouve bien devant une dépense prise à même le budget de l'année suivante. On voit comment le système fonctionne: jusqu'à ce jour, le roi a pourvu aux besoins de l'État colonial en lui expédiant de l'argent pour régler ses comptes; cet argent tombe dans le commerce. De leur côté, les marchands doivent importer des produits français. Ils sont en mesure de payer en monnaie métallique leurs fournisseurs métropolitains. Ils saisissent néanmoins avec empressement l'offre que leur fait l'intendant d'échanger les espèces qu'ils doivent envoyer en France contre des traites sur la Marine parce que l'état de guerre rend extrêmement risqués les transports de numéraire à travers l'Atlantique.

Que va faire la Cour? "Sa Majesté, porte le mémoire du roi de 1692 , a fait payer les 200.000 livres qui ont esté tirez de Canada en lettres de change pour faire partie du fonds des dépenses de la présente année, et Elle trouve bon que le $\mathrm{Sr}$ de Champigny se serve da la voye de ces traittes a l'avenir pour le mesme effect ». Du même souffle, elle annonce que, de son côté, elle prend le parti d'expédier les fonds destinés à la colonie «en vivres et marchandises plustost qu'en argent $\gg^{48}$ Singulière

45 Champigny à Pontchartrain, 12 octobre 1691, ibid., 96.

46 Ibid., 98. 
façon de procéder, contre laquelle Frontenac, trois ans plus tard, élève des plaintes justifiées. Le gouverneur y voit trois inconvénients : d'abord, « il est en quelque façon honteux et fort onereux pour tous les autres marchands que le Roy ayt icy un magasin six fois plus fort qu'aucun des leurs »; de plus, le nouveau système est compliqué, et le profit que le roi tire des marchandises qu'il «debite » dans son magasin ne se révèle pas « aussy grand qu'on lesperoit pour augmenter les fonds de nos depenses extraordinaires »; enfin, le gouvernement français n'envoyant plus de numéraire au pays, celui-ci manque de monnaie, en attendant le jour où il s'en trouvera «entierement denué ».47 Toujours à court de fonds, l'Etat colonial ne trouve pas de meilleur moyen de se tirer d'embarras que de faire, à l'occasion, quelque émission de monnaie de cartes. Jusqu'en 1691 , ces émissions avaient été à très brève échéance. Maintenant, les cartes « courent dans le Commerce » d'une année à l'autre, sans que l'intendant puisse les racheter. ${ }^{48}$

La Cour l'ignorait. Elle se gendarme. Voilà un expédient dangereux, observe Pontchartrain, ajoutant d'un ton sec: «L'intention du Roy est que cela finisse. » Pourquoi ? Il peut surgir «des gens assez hardis» pour contrefaire des cartes ! 49 Forcé d'expliquer comment il a bien pu se porter à cette " extrémité », Champigny s'exécute: «Les provisions de bled que j'ay êté obligé de faire tous les ans, l'envoy de France d'une bonne partie de la solde des troupes en farines et lards qui n'ont êté convertis en leur paye que longtemps après, les dépenses faites au delà des fonds chaque année,... avec le manque de fonds causé par les pertes faites a la mer en 1690, 1691 et 1692 ont été les causes de la monnoye de cartes. ${ }^{50}$ En d'autres termes, quatre motifs ont contraint l'intendant à recourir à l'expédient condamné par son chef: les achats de vivres qu'il a dû effectuer dans la colonie, les pertes encourues en mer, l'insuffisance du budget et le crédit qu'il a fallu consentir aux clients du magasin du roi. Enfin, en 1699, pour qu'il ne soit plus question de cartes, la Cour « a la bonté d'ordonner des remplacemens » de fonds afin d'honorer tou-

47 Frontenac à Lagny, 2 novembre 1695, ibid., 100.

48 Frontenac et Champigny à Pontchartrain, 26 octobre 1696, ibid., 102.

49 Pontchartrain à Champigny, 27 mai 1699, ibid., 104-106.

so Champigny à Pontchartrain, 20 octobre 1699, ibid., 106-108. 
tes les anciennes créances de l'État, dont certaines remontent jusqu'à $1687 . .^{51}$ Ainsi, quand s'ouvre le XVIII ${ }^{e}$ siècle, il ne traîne plus, semble-t-il, aucune carte sur le marché canadien. Au printemps de 1701, le ministre félicite l'intendant de les avoir supprimées «totalement . $^{.2}$

\section{La monnaie de cartes: $1702-1714$}

Si Champigny a fait disparaître les cartes de la circulation, c'est pour les y réintroduire aussitôt. Nous savons que, de concert avec Callières, il en fit une émission en 1702.53 Son successeur, Beauharnais, en fabriqua de nouvelles l'année suivante. ${ }^{54}$ Il en émit encore d'autres en 1704; il les prêta à la Compagnie de la Colonie pour qu'elle pût, ainsi qu'elle y était tenue, payer les traitements et les subventions des personnes et des institutions inscrites à l'état du Domaine. ${ }^{55}$ En 1705 , le gouverneur de Montréal avertissait la Cour que le gouvernement canadien avait déjà jeté trop de cartes dans le commerce: «Il y a icy beaucoup plus de cartes quy [qu'il] ne paroist de fonds pour les retirer, ce quy causera un jour bien du trouble et de la confusion; on en a refusé pour plus de cent mil livres dont le tresorier n'a point voulu donner de lettres de change, ce quy porte un préjudice notable aux marchands et au commerce. ${ }^{56}$ Presque au moment où Ramezay écrivait, le nouvel intendant, Jacques Raudot, publiait une ordonnance destinée à enrayer le discrédit qui commençait

51 Champigny à Pontchartrain, 15 octobre 1700, ibid., 112-114.

52 Pontchartrain à Champigny, 31 mai 1701, ibid., 116. Voir la dépêche de Champigny résumée par Shortt, ibid., 114, note 2 . Comparer à ce qu'écrit pourtant Shortt dans son introduction, ibid., liv.

53 En 1703, le gouverneur de l'Acadie, Brouillan, écrit avoir été «obligé de suivre l'exemple de Canada en faisant faire de la monnoye de cartes 》, ibid., 124. L'année suivante, la Cour ordonne aux administrateurs du Canada de racheter toutes les cartes répandues dans le public, « a commencer par celles qui restent du temps des srs de Callieres et de Champigny 》, ibid., 126. Le gouvernement canadien retira de la circulation pour 5.000 livres de cartes en 1705, ibid., 146.

54 F. H. Hammang, The Marquis de Vaudreuil. New France at the Beginning of the Eighteenth Century (Bruges et Louvain, 1938), 106.

55 Shortt, 1: 132, 148. En 1705, la compagnie devait 194.321 livres à l'Etat, qui les lui avait prêtées en monnaie de cartes. Voir Hammang, op. cit., 107, note 77. En 1717, l'Etat métropolitain remettra à la compagnie une dette évaluée à 154.617 livres en monnaie de cartes, Shortt, 1: 404 .

56 Ramezay à Pontchartrain, 12 octobre 1705, ibid., 140-142. 
à s'attacher à la monnaie de cartes: les tribunaux frapperaient désormais d'une amende de 100 livres les particuliers qui ne voudraient pas recevoir de cartes en paiement de leurs denrées ou de leurs services. ${ }^{57}$

Il se passe donc au Canada quelque chose de nouveau. Dans les quinze dernières années du XVII ${ }^{e}$ siècle, les cartes constituent un expédient. Les administrateurs du Canada les utilisent pour se tirer d'embarras. Ils les rachètent toujours, que ce soit au moyen d'espèces sonnantes ou d'effets qui peuvent produire des espèces. A ce moment, l'Etat a des disponibilités supérieures à la somme des cartes répandues dans le public. Tout autre apparaît la situation dans les premières années du XVIII ${ }^{e}$ siècle. Maintenant, les cartes ne sont plus un expédient, elles deviennent une institution. Dès le début, la guerre de la Succession d'Espagne entraîne le gouvernement colonial à des dépenses que ne prévoient pas les budgets de la Cour. ${ }^{58}$ Sans argent, l'État règle avec des cartes les factures de ses fournisseurs. Par ailleurs, dans les cadres de son budget, l'intendant est autorisé à tirer pour une certaine somme de lettres de change, qu'il distribue aux porteurs de cartes. La colonie dispose ainsi d'une monnaie qui sert au commerce intérieur; cette monnaie sert également au commerce extérieur dès qu'elle peut être convertie en lettres de change. Il en résulte que la monnaie de cartes est désormais gagée sur les traites encaissables à Paris. Que cela, néanmoins, ne nous fasse pas perdre de vue qu'elle représente une dette de l'État métropolitain envers la population canadienne. Sa solidité repose donc, au fond, sur la solvabilité du gouvernement français. Concrètement, la monnaie de cartes joue son rôle à deux conditions: que l'intendant dispose tous les ans d'assez de lettres de change pour racheter les cartes qui lui sont présentées; que les trésoriers généraux de la Marine à Paris honorent les lettres de change que Québec leur envoie.

57 Ordonnance du 24 octobre 1705, ibid., 144-146.

58 En 1702 , se fondant sur les 156.000 livres qu'il avait fallu dépenser pour la guerre l'année précédente, l'intendant demande 125.528 livres pour le même objet; la Cour n'en accorde que 100.000. Beauharnais à Pontchartrain, 11 novembre 1702, AC, C 11A, 20: 109v. 
En 1705, la première de ces deux conditions n'est pas remplie, ce qui explique que l'intendant doive donner cours forcé aux cartes. Deux ans plus tard, c'est la seconde condition qui ne se réalise pas. La guerre obère les finances de la mère-patrie. A bout de ressources, le gouvernement de Louis XIV paie non plus en argent, mais en billets du trésor les traites qui lui sont rapportées. Cet expédient, on le conçoit, a des répercussions désastreuses au Canada. Ce qui ruine le pays, déclare Raudot, «ce sont les lettres de change qui ne sont plus acquittez en France qu'en billets de monnoie sur lesquels il faut perdre cinquante pour cent pour avoir de l'argent comptant $\gg .59$

A cette difficulté, s'en ajoute une autre. Bien qu'il pratique lui-même l'art d'échanger du papier pour du papier, le gouvernement royal ne s'est pas réconcilié avec la monnaie de cartes du Canada. Il ne veut pas que l'intendant en fabrique davantage. Raudot prend le détour de faire faire des billets pour la moitié des fonds que le trésorier de la colonie a en caisse, avec promesse qu'il en sera « remboursé à l'arrivée des vaisseaux 》. Le ministre fait observer avec raison: "Il me paroist que ce sont de nouvelles cartes que vous faites sous un autre nom.» Mais comment le gouvernement canadien peut-il se dispenser de payer de quelque façon ce qu'il achète dans la colonie ? Nécessité d'autant plus grave que ses besoins augmentent, alors que ses disponibilités ne s'améliorent pas. Ces dernières, ce n'est pas la Cour qui va les accroître, elle en prévient l'intendant: «Il n'y a pas eu moyen d'accorder cette année l'augmentation de fonds que vous demandez pour l'achapt de marchandises pour le Canada, et c'est encore beaucoup que Sa Majesté veuille bien continuer les mesmes dépenses pour un pays qui ne luy produit rien et qui luy est fort à charge. ${ }^{60}$ Aussi bien quand, en 1708 , le gouvernement canadien «supplie » Versailles de ne rien retrancher au projet d'état du roi qu'il lui expédie, sa demande ne saurait tomber plus mal. ${ }^{61}$ Fait qui empire encore les choses, l'État métropolitain doit aussi

\footnotetext{
59 Raudot à Pontchartrain, 11 novembre 1707, Shortt, 1: 170. Sur la dépréciation des billets du trésor, voir aussi The Boston News-Letter, 14 juillet 1707.

60 Pontchartrain à Raudot, 6 juin 1708, Shortt, 1: 178.

61 Raudot à Pontchartrain, 14 novembre 1708, ibid., 186-188.
} 
tenir compte des besoins de l'Acadie, menacée plus directement que le Canada par les Anglais, entre les mains de qui elle tombera d'ailleurs bientôt: en cette même année 1708, si le Canada demande pour 54.000 livres de munitions, l'Acadie en réclame pour 81.000 livres. $^{62}$

Précaire en 1708, l'état des finances canadiennes va s'aggraver au cours des années suivantes. En 1710, Québec n'a encore reçu ni le budget de l'année précédente ni celui de l'année en cours; le trésorier général de la Marine n'a envoyé aucun ordre pour 1711 et n'a pas même désigné de commis autorisé à agir en son nom. Faute de fonds, l'intendant n'a pas tiré 40.000 livres de lettres de change sur Paris pour chacun des exercices de 1709 et de 1710. Au reste, depuis que la Marine n'honore plus les lettres de change, les négociants éprouvent à leur égard une «méfiance » bien naturelle. Les effets expédiés de la métropole ont-ils au moins produit des fonds ? Par le passé, ils y ont contribué, mais voilà qu'en 1710 ils ont coûté plus cher au roi que les marchands locaux ne les vendent. ${ }^{63}$ Pourtant, il faut payer la solde des troupes et pourvoir aux autres besoins du pays. Comment y arriver ? En faisant «encore des Cartes ». C'est le parti qu'ont pris Vaudreuil et Raudot. «Il est fascheux, écrit à ce sujet un fonctionnaire français, qu'on ayt esté obligé de faire des cartes. Il y a pourtant une necessité d'en continuer puisqu'on ne leur envoye rien. » C'est ainsi que le gouvernement du Canada lance pour 244.000 livres de cartes en 1710 et pour 450.000 en $1711 .{ }^{64}$

La cause de la pénible situation financière du Canada se trouve dans la détérioration des finances métropolitaines, saignées à blanc par une guerre interminable et malheureuse. Tout,

62 Bégon à Pontchartrain, 11 mars 1708, AC, C 11A, 29: 363.

63 En 1712 , il en sera encore ainsi: «J'ay examiné Lenvoy que vous faites pour la Colonie du Canada et j'y trouve de certaines marchandises augmentées de Cent pour Cent au dessus du prix Courant», Pontchartrain à Beauharnais, 18 juin 1712, $\mathrm{AC}, \mathrm{B} 34: 25 \mathrm{v}-26$.

64 « Memoire sur le Canada》, 1711, AC, C 11A, 31: 197-198; résumé d'une lettre de Raudot, 1711, AC, C 11A, 32: 177-177v; "Bordereau de la nouvelle monnoye de Cartes fabriquées en l'année 1710 », ibid., 284; 《Bordereau de la nouvelle monnoye de Cartes fabriquées en Lannée 1711 », ibid., 7. 
dans ces années noires, témoigne de la pauvreté de l'État français. Par exemple, au début de 1710, l'intendant de Rochefort passe avec Pascaud un marché pour une fourniture de farine aux garnisons de Terre-Neuve et de l'Acadie; le contrat porte que le fournisseur sera payé en poudre tirée des arsenaux, à raison de 70 livres le quintal. Il passe avec un armateur un contrat d'affrètement pour transporter des munitions au Canada; il paye l'armateur en marchandises des magasins du roi. ${ }^{65}$ C'est que le ministère de la Marine n'a pas l'argent nécessaire pour envoyer lui-même l'habituel vaisseau du roi à Québec; il confie cette tâche à des entrepreneurs qu'il paye en nature ou qu'il indemnise en leur réservant le fret que leur procureront les retours des marchands canadiens en France. ${ }^{66} \mathrm{Si}$, après la conquête de l'Acadie, que la Cour exprima tout de suite le désir de reprendre aux Anglais, le gouvernement français n'envoie pas une expédition capable de le faire rentrer en possession de la colonie perdue, ce n'est pas manque de soldats, d'armes ou de vaisseaux, c'est faute de pouvoir défrayer l'entretien d'un corps expéditionnaire. ${ }^{67}$ Après cela, on n'est plus surpris de constater que, loin de se voir en état d'honorer ses obligations canadiennes, la France ne puisse que les laisser s'accumuler au rythme des émissions de monnaie de cartes effectuées dans la colonie.

$\mathrm{Au}$ point où nous en sommes, une question ne peut pas manquer de se poser. Comment les commerçants canadiens, qui montraient de la répugnance à prendre des lettres de change en paiement de leurs fournitures, pouvaient-ils accepter des cartes, alors que celles-ci étaient gagées sur les lettres de change ? C'est que les traites ou bien n'étaient pas acquittées ou bien ne l'étaient qu'en papier très déprécié. Quant aux cartes, du fait qu'elles ne portaient pas d'échéance fixe, on pouvait les garder

65 Résumé d'une lettre de Bégon à Pontchartrain, 2 février 1710, AC, C 11A, 31: 257-258.

66 《Memoire sur le Canada》, 1711, AC, C 11A, 31: 198-199v. Voir Pontchartrain à Bégon, 29 mai 1709, AC, B 30: 125v-126; Pontchartrain à Raudot, 6 juillet 1709, ibid., 143v; Pontchartrain à Bégon, 12 juillet, 31 juillet, 31 décembre 1709, ibid., 187, 194; mémoire à Bégon, 7 juillet 1711, AC, B 33: 108 .

67 Maurepas à Beauharnais, 24 décembre 1710, AC, B 32: 228v-229v; $i d$. à $i d$., 20 février 1711, AC, B 33: 19-21; 30 mars 1711, ibid., 36-37; 6 mai 1712 , ibid., 52. 
en caisse; un jour, la guerre finirait; le crédit de la Marine se rétablirait: alors, il serait temps d'échanger les cartes thésaurisées pour de bonnes lettres de change. ${ }^{68}$ En somme, acquérir de la monnaie de cartes, c'était miser sur l'avenir.

N'en concluons pas que la valeur des cartes restait d'une solidité à toute épreuve. En 1711, Vaudreuil représente à la Cour la nécessité d'acquitter les lettres de change des années précédentes «afin que cela donne un peu de crédit à nos cartes》; sinon, déclare-t-il, «ce pays va tomber dans la derniere des miseres ».69 La réponse du ministre de la Marine sera décourageante:

Plusieurs personnes du Canada m'ont représenté cette année et sur tout $\mathrm{M}^{\mathrm{rs}}$ de Vaudreuil et Raudot quil sera impossible a l'avenir de trouver les moyens de faire subsister les troupes et de fournir aux depenses ordinaires et extraordinaires si les Tresoriers generaux nacquittent les lettres de change a leur echeance, et que la grande quantité de cartes qu'on a fabriquées dans le pays les met dans le decry, ce qui fait augmenter les marchandises du quadruple parce que les negocians ne peuvent pas sen faire [sic] et quelles ne leur produisent que des lettres de change qui ne sont pas acquittées ce qui les ruine en Interests dargent et fait souffrir generalement toute la Colonie. Je conviens de la Vérité de tous ses faits...

On ne saurait mieux décrire l'état des finances du Canada. Le gouvernement français connaît le mal dont «souffre» la colonie. Connaît-il aussi bien le remède qu'il serait urgent d'y appliquer ? A la vérité, il n'y en aurait qu'un: que le roi payât ses dettes. Or, répond le ministre, non seulement en est-il incapable, mais même la paix ne le mettra pas en état d'honorer toutes ses obligations, «dont les cartes du Canada ne font qu'une partie »; tout ce que le roi «pourra faire alors sera de payer le Courant ». Dans ces conditions, qu'adviendra-t-il des cartes ? Il se peut qu'elles tombent toutes «en pure perte a ceux qui les

68 Voir Shortt, 1: 208, note 2; délibération de Vaudreuil, Raudot et Monseignat, 1er octobre 1711, ibid., 210-212; Vaudreuil et Raudot, ordonnance du 25 octobre 1711, ibid., 212-214.

69 Vaudreuil à Pontchartrain, 8 novembre 1711, ibid., 218. 
auront et qui par leur opiniatreté Voudront les garder absolument ». Aussi faut-il que le gouverneur et l'intendant cherchent «des expédients pour en diminuer le nombre». Il est essentiel de les retirer de la circulation. ${ }^{70}$

L'année précédente, en confiant à Bégon l'intendance de la Nouvelle-France, la Cour avait fait au fonctionnaire la même recommandation, en l'autorisant cependant avec plus de réalisme que d'esprit de suite à émettre d'autres cartes quand il s'y verrait absolument forcé, «ce qu'il doit entendre par raport a la difficulté qu'il trouvera de faire tirer des lettres de change sur les tresoriers generaux de la marine pour remplir le fonds que $\mathrm{Sa} \mathrm{Ma}^{\text {té }}$ fait annuellement pour le Canada ». En d'autres termes, la consigne avait été de racheter les cartes, mais aussi d'en répandre d'autres pour tirer des Canadiens les produits et les services dont l'État colonial aurait besoin et que la métropole ne pourrait payer qu'en lettres de change auxquelles les habitants préféraient les cartes. Il faut ajouter que la Cour ne savait pas combien il circulait de cette monnaie dans la colonie, puisqu'elle demandait à Bégon «un Estat juste année par année dans lequel il marquera [les cartes] qui ont esté retirées, ce qui en reste dans le commerce, par qui elles sont deuës, et sur quels fonds elles doivent estre remboursées $\gg .{ }^{71}$

Combien y avait-il donc de cartes au Canada ? En 1713, Vaudreuil et Bégon avouent qu'il faudrait les retirer «pour connoitre pour quelle somme il y en a dans le public $\gg .{ }^{72}$ Le registre contenant les procès-verbaux des émissions et des retraits depuis 1702 jusqu'à 1711 avait disparu dans l'incendie du palais de l'intendant, le 5 janvier 1713. Le contrôleur d'Aigremont avait bien, avant l'accident, dressé d'après ce registre un état de toute la monnaie de cartes lancée sur le marché. Mais soit que cet état ait été fautif, soit que le registre lui-même ait été mal tenu, la quantité de cartes répandue dans la colonie ne paraissait pas correspondre à celle que le contrôleur avait calculée. ${ }^{73}$ Selon

70 Maurepas à Vaudreuil et à Bégon, 26 juin 1712, ibid., 218-222.

71 « Memoire pour servir d'Instruction au Sr Begon》, 7 juillet 1711, AC, B 33: $99 \mathrm{v}-100$.

72 Lettre du 15 novembre 1713, AC, C 11A, 34: 22.

73 Aigremont à Maurepas, 15 novembre 1713, Shortt, 1: 254-256. 
Ruette d'Auteuil, il existait en 1712 pour plus de 1.300 .000 livres de cartes, et le gouvernement en aurait fabriqué encore pour un demi-million avant la fin de $1713 .{ }^{74}$ De 1711 à 1714 , Bégon savait qu'il s'en était fait pour 948.600 livres et il en avait retracé pour 970.312 livres des émissions antérieures. ${ }^{75}$ Quand il sera question de racheter cette monnaie, on en ramènera le montant à 1.600 .000 livres.

Encore une fois, une pareille somme représente la dette du roi envers la population canadienne. Celle-ci, bien entendu, n'abandonne pas ses créances. L'intendant énumère les conséquences de cette situation:

... Si les fonds ordonnés pour les dépenses de l'année prochaine ne sont pas remis en ce pays, soit en faisant payer les lettres de change qui seront tirées sur $\mathrm{M}^{\mathrm{rs}}$ les Tresoriers, ou par l'Envoy de marchandises achettées en France a leur juste valeur, on ne sera ny en estat d'habiller et de faire subsister les troupes, ny de donner aux sauvages tous les presens qu'il convient de leur faire pour les maintenir dans nos Interests, ny d'envoyer de partis contre les Anglois, parce que les magasins du Roy estant tout a fait dégarnis, tout ce qu'on achette icy qui ne cousteroit au Roy, s'il estoit envoyé de France au prix courant, que 25.000 livres revient icy a Sa Majesté a 100.000 livres par la necessité dans laquelle on est de prendre a present chez les marchands tout ce qu'on a besoin; ainsi par cette misere les fonds ordonnés par Sa Majesté se trouvent diminués des trois quarts. ${ }^{76}$

Comme l'Etat ne peut pas assurer les services essentiels sans disposer d'un minimum de ressources, Vaudreuil emprunte aux négociants canadiens $\mathbf{1 5 0 . 0 0 0 ~ l i v r e s , ~ q u ' i l s ~ l u i ~ c e ̀ d e n t ~ e n ~ c a s - ~}$ tor, à condition qu'il leur rembourse le quart de cette valeur en lettres de change sur le Domaine d'Occident, alors rattaché aux fermes générales. L'ennui est que l'agent des fermiers du Domaine ne peut pas tirer de traites à volonté: pour faire face à

74 « Mémoire à Son Altesse Royale Monseigneur le Duc d'Orléans ... sur l'état présent du Canada », 12 décembre 1715, RAPQ (1922-23), 65.

75 Bégon à Pontchartrain, 12 novembre 1714, Shortt, 1 : 282-284.

76 Bégon à Pontchartrain, 12 novembre 1712, AC, C 11A, 33: 128v-129. 
cette obligation supplémentaire, il doit laisser en souffrance les réclamations justifiées d'une grande partie des institutions et des fonctionnaires inscrits pour des subventions régulières sur l'état du Domaine. ${ }^{77}$ Cet emprunt ne suffit pas. Le gouvernement en contracte d'autres. Quand il est question de les acquitter, les administrateurs offrent aux créanciers des lettres de change, que ceux-ci refusent. Il leur faut faire encore des cartes. ${ }^{78}$

A côté des cartes, dont la valeur baisse tous les jours, il n'y a plus dans le commerce canadien que des lettres de change sur la compagnie française du castor et sur le Domaine d'Occident. Encore solides, ces deux catégories de traites permettent aux négociants du pays de faire des achats en France, mais des achats fort limités par rapport à ceux qu'ils pourraient effectuer si les lettres de change sur la Marine étaient négociables. ${ }^{79}$ C'est précisément ce qui fait la gravité de la crise dans laquelle la colonie se débat. Et c'est là qu'on voit l'importance du rôle de l'État de l'État métropolitain et de l'État colonial - dans le développement économique du Canada.

La Cour ne peut pas se dispenser de proposer à la colonie une solution. On le sait déjà, de solution, elle n'en voit qu'une: le retrait des cartes. Elle s'imagine pouvoir l'opérer sans qu'il lui en coûte grand'chose pour l'instant. En 1712, la Cour a recommandé aux administrateurs de la colonie «d'exciter les marchands qui ont considerablement de ces cartes a prendre des Lettres de change quils convertiroient icy en billets des Tresoriers generaux de la marine qui sont receus en rentes sur la Ville de Paris ou sur les generalités du Royaume ${ }^{80}$ En d'autres termes, il s'agissait de convertir les cartes en obligations municipales et provinciales. La proposition était si peu alléchante que Vaudreuil et Bégon n'osèrent même pas la communiquer aux Canadiens, de peur de faire baisser encore la valeur des cartes et

\footnotetext{
${ }_{77}$ Monseignat à Maurepas, 9 novembre 1712, ibid., 205v-206.

78 Vaudreuil et Bégon à Pontchartrain, 12 novembre 1712, Shortt, 1: 232-234.

79 Vaudreuil et Bégon à Pontchartrain, 15 novembre 1713, AC, C 11A, 34: $9 \mathrm{v}$.

80 Maurepas à Vaudreuil et à Bégon, 26 juin 1712, Shortt, 1: 220. Voir les explications données $i b i d$., notes 1 et 2 .
} 
d'imprimer ainsi un nouvel élan à l'inflation. ${ }^{81}$ C'est pourtant la même solution que la Cour préconise encore en 1713. Cette fois, en vue d'induire les détenteurs de cartes à les rapporter au trésor pour opérer la conversion indiquée, elle autorise le gouverneur et l'intendant à ordonner une diminution annuelle de cinq ou mieux de dix pour cent sur la valeur des cartes en circulation. ${ }^{82}$ L'intendant ne pourra rien faire à cet égard en 1713 , vu que le vaisseau qui devait lui apporter la dépêche du ministre ne se rendit pas au Canada.

Suppression des cartes: $1714-1720$

En 1714, le gouvernement métropolitain commence par donner des ordres analogues à ceux de l'année précédente, puis il adopte la proposition que Bégon lui avait soumise de racheter toute la monnaie de cartes à la moitié de sa valeur nominale. Ce rachat doit s'effectuer en cinq ans. Tous les ans, l'intendant fera rentrer 320.000 livres de cartes, en échange desquelles il délivrera aux porteurs 160.000 livres de traites. Celles-ci seront faites à l'ordre du trésorier général de la Marine, qui les acquittera en argent. Quant aux dépenses courantes, l'intendant les réglera en lançant d'autres émissions de cartes qui, elles, ne seront pas sujettes à la dévaluation. ${ }^{83}$ "La pitoyable espérance, raconte Auteuil, de ne perdre que la moitié de son bien lorsqu'on estimait perdre le tout ne laissa pas de ranimer le courage des marchands $\gg .{ }^{84}$ Un fonctionnaire exprime un point de vue différent: «Les Négociants, et les habitants proffiteront considérablement de la diminution de la moitié de la valeur des cartes; Les premiers vendants leurs marchandises beaucoup au delà du double de l'année dernière; Et les autres leurs denrées a proportion; les artisants et les ouvriers augmentent leur travail sur le mesme pied; il n'y a que les pauvres, et ceux qui sont aux appointements du Roy qui souffrent notablement et qui ne peuvent se

81 Ibid., 232. Les cartes, dit en 1713 Monseignat, « sont tombées dans un avilissement qu'on ne peut exprimer $\gg$. En conséquence, les prix montent, et \& les habitants vendent leyrs denrées a proportion que les marchands vendent leurs marchandises », AC, C 11A, 34: 109v.

82 Shortt, 1: 238-252.

83 Ibid., 266-270.

84 RAPQ (1922-23), 66. 
donner le quart du nécessaire. ${ }^{85}$ C'est ce que confirme Vaudreuil: à son dire, les manœuvres, qui ne gagnaient autrefois qu'une livre par jour, en touchent maintenant trois. ${ }^{86}$ Les prix, semble-t-il, s'ajustent au cours de la monnaie. ${ }^{87}$

Dans l'ensemble, les Canadiens, les commerçants en tête, acceptent sans hésiter que l'État rachète leurs cartes aux conditions qu'il a fixées. Aucun témoignage n'est plus éloquent à cet égard que le fait suivant. En 1714, les porteurs de cartes marquent tant d'empressement à se rendre à l'invitation de l'État que Bégon peut non seulement racheter pour 320.000 livres de monnaie ainsi qu'il était convenu, mais en retirer encore pour 316.110 livres, en échange de quoi il donne des traites au montant de 160.000 livres payables en 1715 et de 158.055 livres payables en $1716 .^{88}$ Cette anticipation soulageait le marché canadien de 636.110 livres de cartes et indiquait en même temps à quel point se portait la confiance du public envers les lettres de change.

Cette confiance allait tout de suite éprouver un choc. Quand les lettres de change du Canada furent présentées au trésorier général de la Marine, celui-ci se déclara incapable de les acquitter, «faute de fonds ${ }^{89}$ Cependant, promit Pontchartrain, « elles seront payées sinon a l'echeance precise au moins quelque tems apres, il y a eu du retardement au payement de celles de cette année il a eté causé par la rareté des Especes causée en parti par le derangement qu'il y a depuis quelques mois dans le Commerce et le nombre considérable de Banqueroutes qu'il y a eu dans le Royaume ${ }^{90}$ En attendant, malgré le ton rassurant de la Cour, les porteurs de lettres de change canadiennes poursuivaient le trésorier général Gaudion, qui fut «condamné a etre arreté, ce qui cause un scandale effroyable », déplorait le minis-

85 Monseignat à Pontchartrain, 8 novembre 1714, Shortt, 1: 282.

86 Ibid., 272.

87 Pour savoir exactement quel fut le mouvement des prix en ces années de crise, il faudrait, bien entendu, procéder à une étude spéciale du sujet. 290.

88 Pontchartrain à Nointel, 9 janvier 1715, Shortt, 1: 286. Voir ibid.,

89 Pontchartrain à Desmaretz, 2 juillet 1715, ibid., 298.

90 Pontchartrain à Bégon, 10 juillet 1715, ibid., 300 . 
tre. ${ }^{91}$ Dans la colonie, habitants et négociants, suffoqués par cette nouvelle, «murmurent Les uns et les autres au dessus de toute Expression $\gg .{ }^{92}$ Le commerce s'arrêta, les ruraux refusant de céder leur blé pour de la monnaie de cartes, «sur ce que les negociants disoient qu'ils n'en recevroient plus en payement de leurs marchandises, lesquelles ils ne vendroient plus qu'en troc de pelleteries $\gg .{ }^{93}$

Dans ces conditions, la froideur des commerçants n'a rien d'étonnant lorsque Bégon, en 1715, essaie de racheter, à la moitié de sa valeur, une autre tranche de 320.000 livres de cartes; on ne lui en apporte que pour 122.000 livres, en retour desquelles il distribue 61.000 livres en lettres de change payables en $1717 .{ }^{94}$ En 1716, la Cour annonce que le trésorier général de la Marine a payé pour 120.000 livres de traites du Canada encaissables en mars 1715. Il lui reste donc à honorer, au cours de 1716, environ 200.000 livres de lettres déjà échues; quant aux 61.000 livres payables en mars 1717, le ministre assure qu'elles «seront acquittées a leur Echeance de mesme que celles qui seront tirées a l'avenir Jusques a l'Extinction Entiere des Cartes $\gg .^{95}$

En 1717, la situation sera la suivante. Bégon a racheté depuis quatre ans 1.155.380 de monnaie de cartes, en paiement de quoi il a tiré sur Paris pour 577.690 livres de lettres de change; la Marine en a acquitté pour 300.000 livres. Il ne devrait rester qu'environ 450.000 livres en cartes sur le marché canadien. Il en reste cependant encore, assure-t-on, pour 1.300 .000 livres. $^{96}$ Pourquoi ? Parce que l'État métropolitain, incapable de faire face à ses obligations passées, éprouve, bien entendu, les pires difficultés à pourvoir aux dépenses courantes de la colonie. Avec quoi l'intendant paye-t-il ses comptes ? Toujours avec des cartes. La Cour voudrait que Bégon détruisît celles qu'il rachète et en émît d'autres, différentes des premières. Les finances du pays, réplique le magistrat, sont déjà assez embarrassées par une seule espèce de cartes sans en lancer d'autres d'une seconde espèce;

91 Pontchartrain à Desmaretz, 17 juillet 1715, ibid., 302.

92 Ramezay à Pontchartrain, 28 octobre 1715, AC, C 11A, 35: 96.

93 Bégon à Pontchartrain, 7 novembre 1715 , ibid., $135 \mathrm{v}$.

94 Shortt, 1: 324.

95 Ibid., 352.

96 Ibid., 384. 
du reste, « ces cartes d'une marque nouvelle auroient fait tomber toutes les autres dans un si grand mepris qu'on n'en auroit point voulu ».97 Aussi les mêmes cartes seront-elles rachetées plus d'une fois.

Ce qui rend cette opération inévitable, c'est la lenteur avec laquelle s'opère leur convertissement. Non pas que le gouvernement royal ne veuille pas aller vite en besogne. Au contraire, une «Déclaration du Roy» du 5 juillet 1717 témoigne de sa hâte d'en finir. Cette législation autorise une dernière émission de cartes pour payer les dépenses du second semestre de 1716 et du premier de 1717. Ensuite, il sera absolument interdit au gouverneur et à l'intendant «de faire fabriquer aucune monnoye de cartes ». Le cours officiel de cette émission sera de $50 \%$, à compter du jour de l'enregistrement de la «Déclaration 》. A commencer du même jour, toutes les cartes devront rentrer au trésor colonial et être converties en traites payables en France par tiers, en mars 1718, 1719 et 1720 . Les Canadiens auront jusqu'à l'automne de 1718 pour se départir de leur monnaie, après quoi celle-ci perdra toute valeur. Elle sera donc liquidée en 1720. Telle est, en 1717, l'intention de la Cour, désireuse de mettre un terme aux «inconvenients que la monnoye de cartes cause dans nostre colonie de Canada $\gg .98$

Seulement, on le voit une fois de plus, il y a loin de l'intention aux réalisations. En 1718, on n'est guère plus avancé que l'année précédente. D'abord, il reste encore à acquitter la moitié des lettres de change tirées en 1716 et payables en 1717. De plus, le trésorier général de la Marine n'est en mesure d'accepter ni les lettres de change de 1716 payables en 1718 ni les traites de 1717 faites en vertu de la «Déclaration» de la même année.99 En conséquence, le roi ordonne de prolonger d'un an l'existence des cartes. Enfin, en 1719, il peut apprendre aux Canadiens qu'il a fait acquitter les lettres de change qui ont servi au rachat des cartes; celles-ci s'en trouvent donc supprimées. ${ }^{1}$ Quand s'ouvre l'année 1720 , la monnaie de cartes a disparu du marché cana-

97 Ibid., 318.

98 Ibid., 398-400.

99 Ibid., 428.

1 Ibid., 460. 
dien. En comptant toutes les cartes fabriquées depuis 1702, y compris celles qui avaient été lancées ou remises en circulation après 1714, l'intendant calculait qu'il en avait couru pour la valeur de 3.355.115 livres. ${ }^{2}$ Pour saisir l'importance de cette somme, il faut réfléchir qu'elle égale à peu près celle des dix budgets accordés à la colonie entre 1711 et 1720 ou encore celle des dépenses de sept ans (1714-1720).

Si l'Etat métropolitain est parvenu tant bien que mal à payer cette dette - énorme pour le Canada et pour l'époque c'est grâce aux ressources artificielles que lui ont procurées, un moment, le magicien John Law et sa Banque générale. Du même coup, le roi a aboli dans la colonie une monnaie dépréciée. Dépréciée, mais utile, on s'en rend maintenant compte. Au Cana$\mathrm{da}$, il ne doit plus circuler que de l'argent. D'où vient-il ? Naturellement, des fonds que le roi ordonne pour les dépenses du pays. Mais Sa Majesté ne délie pas facilement sa bourse. Il serait nécessaire, disent le gouverneur et l'intendant, que le trésorier général de la Marine fît dans la colonie ses remises en espèces, ne retenant en France que deux articles: les appointements des fonctionnaires qui désirent toucher à Paris, par procuration, ce qui leur est dû et le prix des munitions et marchandises envoyées des ports métropolitains aux magasins du roi à Québec. Il faudrait aussi que le fermier du Domaine acquittât en argent l'état des charges. Ce n'est pas ce qui se pratique. Aussi le Canada souffre-t-il bientôt d'une grave disette de numéraire, dont les répercussions sont extrêmement sensibles sur le commerce intérieur et sur le commerce extérieur. Il ne se trouve plus dans le pays qu'environ 100.000 livres en espèces, somme infime si on la compare aux $\ll 3.000 .000$ de livres de monnoye de cartes qu'il pouvoit y avoir et dont chacun se servoit utilement pour son Commerce ». 3 Ce que les administrateurs coloniaux ne semblent pas voir encore, c'est que les excédents annuels de dépenses, qui

2 \& Estat abregé de toute la monnoye de Carte fabriquée en Canada par $\mathrm{M}^{\mathrm{rs}}$ les Gouverneurs generaux et Intendans depuis 1702 . Jusques en 1717. laquelle a esté brulée Suivant les proces Verbaux cy apres », AC, C 11A, 44:217-217v.

3 Vaudreuil et Bégon au Conseil de Marine, 6 novembre 1720, AC, C 11A, 42: 101-105. 
se traduisaient naguère par une augmentation du volume des cartes en circulation, ne peuvent plus donner lieu désormais qu'à des anticipations, et celles-ci n'ont pas l'effet d'ajouter de nouvelles devises à la monnaie disponible pour le commerce intérieur. Dans cette conjoncture, l'apparition d'instruments scripturaires est inévitable. L'État colonial paye les traitements qu'il verse et les achats qu'il effectue en ordonnances de paiement; pressés par des besoins semblables, les négociants font leurs affaires au moyen de «billets particuliers » qui circulent vaille que vaille.

Après comme avant 1720 , les mêmes causes ont les mêmes effets. En 1728, les administrateurs du pays rapportent: «Tout le public nous a fait des representations par des requêtes pour faire de la monnoye de carte que l'on feroit retirer a l'arrivée du vaisseau du Roi; le commerce des billets particuliers est fort dangereux, et donne lieu a une usure epouvantable. ${ }^{4}$ Quelques mois auparavant, la Cour avait prévenu le gouvernement canadien qu'il ne recevrait pas un sou. L'état du roi fut expédié à Québec comme d'ordinaire; il s'élevait à 373.000 livres, outre 60.000 livres à compte des dépenses de 1729. Mais, expliquait Maurepas, ces deux sommes «se trouvant consommées au moyen des fonds remis d'avance en 1727, des Lettres de Change tirées sur l'exercice de 1728, et des munitions et marchandises envoyées de Rochefort, il n'y aura pas un sol a remettre en especes, dont je suis bien faché; il en sera de même tous les ans si l'on continue de tirer [au Canada] des lettres de change pour des sommes aussi considerables que les années precedentes, et il est bien a souhaiter que l'on puisse les reduire au plus a 200.000 livres pour qu'on soit en etat d'envoyer quelque argent $\gg .^{5}$

En d'autres termes, au lieu d'avoir pour conséquence la création d'une monnaie fiduciaire reconnue comme telle, les inévitables excédents de dépenses ont pour résultat de tarir à sa source, dans la métropole, le crédit de la colonie. A quoi cette politique aboutit concrètement, les négociants canadiens le

4 Beauharnais et Aigremont à Maurepas, 11 novembre 1728, AC, C $11 \mathrm{~A}, 50: 120$. 436-436v.

5 Maurepas à Beauharnais et à Dupuy, 11 mai 1728, AC, C 11A. 50: 
disent lorsqu'ils se représentent «chargez d'une quantité de marchandises dont ils ne peuvent se procurer aucune vente qui resteront invendues dans Leurs magazins jusqu'a L'année prochaine sans qu'ils puissent esperer qu'il s'en fasse aucune consommation, ce qui Causera Infailliblement leur Ruine Et celle du Commerce de la Colonie ... Le Defaut de Circulation d'Especes dans la Colonie est la seule source de La Cessation du commerce. $\gg 6$

(à suivre)

Université de Montréal

GuY Frégault

6 Requête à Beauharnais et à Hocquart, 1728, ibid., 77-78. Ce document porte la signature de 24 négociants. 